\title{
Distinct Changes Occur in the Human Breast Milk Microbiome Between Early and Established Lactation in Breastfeeding Guatemalan Mothers
}

\section{OPEN ACCESS}

Edited by:

Ana Rivas,

University of Granada, Spain

Reviewed by:

Rosa Del Campo,

Ramón y Cajal Institute for Health

Research, Spain

Margarita Aguilera,

University of Granada, Spain

*Correspondence:

Kristine G. Kosk

kristine.koski@mcgill.ca

Specialty section This article was submitted to

Food Microbiology,

a section of the journal

Frontiers in Microbiology

Received: 29 April 2020 Accepted: 21 January 2021

Published: 12 February 2021

Citation:

Gonzalez E, Brereton NJB, Li C,

Lopez Leyva L, Solomons NW,

Agellon LB, Scott ME and Koski KG

(2021) Distinct Changes Occur

in the Human Breast Milk Microbiome

Between Early and Established

Lactation in Breastfeeding

Guatemalan Mothers.

Front. Microbiol. 12:557180.

doi: 10.3389/fmicb.2021.557180

\author{
Emmanuel Gonzalez ${ }^{1,2}$, Nicholas J. B. Brereton ${ }^{3}$, Chen Li', Lilian Lopez Leyva4, \\ Noel W. Solomons ${ }^{5}$, Luis B. Agellon ${ }^{5}$, Marilyn E. Scott ${ }^{6}$ and Kristine G. Koski*
}

${ }^{1}$ Canadian Centre for Computational Genomics (C3G), Department of Human Genetics, McGill University, Montréal, QC, Canada, ${ }^{2}$ Microbiome Research Platform, McGill Interdisciplinary Initiative in Infection and Immunity (MI4), Genome Centre, McGill University, Montréal, QC, Canada, ${ }^{3}$ Institut de Recherche en Biologie Végétale, Université de Montréal, Montréal, QC, Canada, ${ }^{4}$ School of Human Nutrition, Mc Gill University, Ste-Anne de Bellevue, QC, Canada, ${ }^{5}$ Center for Studies of Sensory Impairment, Aging and Metabolism (CeSSIAM), Guatemala City, Guatemala, ${ }^{6}$ Institute of Parasitology, McGill University, Ste-Anne de Bellevue, QC, Canada

Human breast milk contains a diverse community of bacteria, but as breast milk microbiome studies have largely focused on mothers from high income countries where few women breastfeed to 6 months, the temporal changes in the breast milk microbiome that occur during later lactation stages have not been explored. For this cross-sectional study, microbiota from breast milk samples of Mam-Mayan mothers living in eight remote rural communities in the Western Highlands of Guatemala were analyzed. All mothers delivered vaginally and breastfed their infants for 6 months. Breast milk from 76 unrelated mothers was used to compare two lactation stages, either "early" (6-46 days postpartum, $n=33$ ) or "late" (109-184 days post-partum, $n=43)$. Breast milk microbial communities were assessed using $16 \mathrm{~S}$ ribosomal RNA gene sequencing and lactation stages were compared using DESeq2 differential abundance analysis. A total of 1,505 OTUs were identified, including 287 which could be annotated as putative species. Among several maternal factors, lactation stage explained microbiome variance and inertia in ordination with the most significance $(p<0.001)$. Differential abundance analysis identified 137 OTUs as significantly higher in either early or late lactation. These included a general shift from Staphylococcus and Streptococcus species in early lactation to Sphingobium and Pseudomonas species in late lactation. Species enriched in early lactation included putative commensal bacteria known to colonize the infant oral and intestinal tracts whereas species enriched in late lactation had a uniform functional trait associated with aromatic compound degradation. Differentially abundant species also included several species which have not previously been reported within breast milk, such as Janthinobacterium agaricidamnosum, Novosphingobium clariflavum, Ottowia beijingensis, and Flavobacterium cucumis. These discoveries describe temporal changes to the breast milk microbiome of healthy Guatemalan mothers from early to late 
lactation. Collectively, these findings illustrate how studying under-represented human populations might advance our understanding of factors that modulate the human milk microbiome in low and middle income countries (LMIC).

Keywords: 16S rRNA gene, human breast milk, microbiome, lactation stage, metagenomics 16S

\section{INTRODUCTION}

Human breast milk is a source of macronutrients and micronutrients essential for infant nutrition (Cunningham et al., 1991; Walker, 2010; Andreas et al., 2015). Research has established that breast milk also provides infants with a continuous source of commensal and potentially beneficial bacteria which can act to inoculate the infant respiratory and gastrointestinal tracts (Fernández et al., 2013; McGuire and McGuire, 2017). Predominant genera commonly reported include Staphylococcus, Streptococcus, Pseudomonas, Cutibacterium (formally Propionibacterium), or Bifidobacterium (Jeurink et al., 2012; Fitzstevens et al., 2017). Recently, metagenomic sequencing studies have begun to characterize the diverse bacterial species in breast milk to better understand how this process might be associated with infant and long-term health (Biagi et al., 2017; Ruiz et al., 2019; Sakwinska and Bosco, 2019).

Several maternal factors are thought to modify bacterial communities in human breast milk. One study reported differences in bacteria (observed at the genera level) related to stage of lactation, maternal BMI and mode of delivery (CabreraRubio et al., 2012), but other studies have reported little or no differences in relative abundance of genera due to maternal age, parity, mode of delivery, or infant sex (Urbaniak et al., 2016; Williams et al., 2017). Given the advancements in resolution of microbial barcoding technologies (Knight et al., 2018; Bolyen et al., 2019; Gonzalez et al., 2019), coupled with the need to identify the factors that influence breast milk microbiota (McGuire and McGuire, 2017), the purpose of the study was to determine if human milk microbiota differed by maternal age, BMI, parity, breastfeeding practices and lactation stage. Breast milk samples were collected from unrelated lactating mothers living in the remote Western Highlands of Guatemala during early (6-46 days postpartum) or established lactation (109-184 days postpartum) as previously described (Wren et al., 2015).

\section{MATERIALS AND METHODS}

\section{Study Site and Participants}

This cross-sectional study was part of collaboration between McGill University and the Center for Studies of Sensory Impairment, Aging and Metabolism (CeSSIAM) in the Republic of Guatemala. Field studies were conducted in eight rural Mamspeaking communities of the San Juan Ostuncalco region in Guatemala (Chomat et al., 2015) between June 2012 and January 2013. Lactating mothers were identified and invited to participate by community health workers. Individuals who had been treated with antibiotics were excluded. All mothers delivered vaginally and breastfed their infants. Samples from participating mothers with infants aged from 6 to $46(n=33)$ and 109-184 $(n=43)$ days postpartum were used to compare the milk microbiome during early and late lactation. Breastfeeding practices were also compared. Exclusively breastfeeding was defined as infants receiving only breast milk; predominant breastfeeding was defined as infants who breastfed but also received water, most commonly as "agüitas" (herbal teas), and mixed breastfeeding was defined as infants who breastfed but were also given solid foods in addition to aguitas (Wren et al., 2015).

\section{Breast Milk Sample Collection}

Prior to sample collection, the nipple and areola of the breast were cleaned with $70 \%$ ethanol. Breast milk samples were collected during a $3 \mathrm{~h}$ time window in the morning from the breast (not recently used for breastfeeding) via full manual expression by a trained midwife. Milk was collected into sterile $60 \mathrm{ml}$ plastic vials and immediately stored on ice. Samples were partitioned into $15 \mathrm{ml}$ tubes at the field laboratory $\left(-30^{\circ} \mathrm{C}\right)$ prior to transfer on dry ice to $\mathrm{McGill}$ University where they were stored at $-80^{\circ} \mathrm{C}$ (Li et al., 2016).

\section{S rRNA Gene Amplification, Sequencing, and Bioinformatics}

DNA extraction was performed using $1 \mathrm{ml}$ of milk with DNeasy Blood and Tissue mini kit from Qiagen according to the manufacturer's protocol by Genomic Quebec Innovation Centre. For PCR, a target region of $\sim 526$ bp based on the Escherichia coli 16S rRNA gene covering the V1-V3 was amplified with the primers 27F (3'-AGAGTTTGATCCTGGCTCAG-5') and 533R (3'-TTACCGCGGCTGCTGGCAC-5') (Cabrera-Rubio et al., 2012; Mediano et al., 2017; Lackey et al., 2019). Amplification used the following conditions: initial denaturation $94^{\circ} \mathrm{C}$ for $2 \mathrm{~min}$, denaturation $94^{\circ} \mathrm{C}$ for $30 \mathrm{~s}$, annealing $58^{\circ} \mathrm{C}$ for $30 \mathrm{~s}$, extension $72^{\circ} \mathrm{C}$ for $30 \mathrm{~s}$, final extension $72^{\circ} \mathrm{C}$ for $7 \mathrm{~min}, 4^{\circ} \mathrm{C}$ hold, over 35 cycles. Amplification was conducted by the Genomic Quebec Innovation Centre at McGill University and sequencing was performed using the Illumina MiSeq platform. Reagent controls were below the detection limit used by Genomic Quebec Innovation Centre for quality assurance. The Anchor pipeline (Gonzalez et al., 2019) was used to process amplicon sequences. Briefly, Mothur (Schloss et al., 2009) was used to align and dereplicate sequences before high count OTU selection at a count threshold of 36 across all samples. NCBI 16S rRNA RefSeq, NCBI non-redundant nucleotide, SILVA, and the Ribosomal Database Project (RDP) databases were used to annotate OTUs using BLASTn with criteria of $>99 \%$ for identity and coverage. When a BLASTn return had 100\% identity and coverage hits across multiple database, priority was given to NCBI $16 \mathrm{~S}$ rRNA 
RefSeq due to the high standard of curation. Low counting amplicons ( $<36$ counts) were binned to high-count OTUs at a lower threshold of $>98 \%$ identity/coverage. Multiple, equally good (highest identity/coverage), annotation was retained and reported. All annotation, and in particular species calls, should be considered putative even when sharing $100 \%$ sequence identity to a single species due to database errors.

Alpha diversity of breast milk samples was measured using Shannon and inverse Simpson indices within Phyloseq R package (McMurdie and Holmes, 2013). Beta diversity was estimated based on variation stabilization normalized counts (rlog) using Bray-Curtis dissimilarity and the Constrained Analysis of Principal Coordinates (CAP) ordination method. Dispersion ellipses were drawn using veganCovEllipse function from Vegan package in R (Oksanen et al., 2017). Significant distance was evaluated between the groups using non-parametric analysis of similarities (ANOSIM) on normalized counts based on Bray distances (Vegan package). To characterize differentially abundant taxonomic units between groups of samples, parametric models developed in transcriptomics perform well when applied to microbiome biomarker data (uneven library sizes, sparsity, and sample representativity) (Jonsson et al., 2016; Weiss et al., 2017; Brereton et al., 2019; Gonzalez et al., 2019; Minerbi et al., 2019). DESeq2 procedure (Love et al., 2014a) was used to calculate differentially abundant taxonomic units. Taxonomic units tested with a false discovery rate (FDR or the expected proportion of false positive findings) $<0.1$ were considered significant (Anders et al., 2013; Love et al., 2014b, 2015; Gonzalez et al., 2019). Pearson's correlation was calculated between rlog normalized OTU counts. Correlation matrices were sorted based on hierarchical clustering of OTUs and represented using $\mathrm{R}$ package corrplot (Wei and Simko, 2017).

\section{RESULTS}

\section{Characterization of Guatemalan Mothers}

Participating mothers were randomly selected from 8 remote Mam-Mayan villages in the Western Highlands of Guatemala: Los Romero (28\%), Buena Vista (25\%), Los Alnonzo (13\%), and $<10 \%$ from each of Espumpujá, Los Lopez, Los Perez, La Unión, and La Esperanza. Population characteristics by lactation stage are summarized in Table 1 . With regards to breastfeeding practices, $86 \%$ exclusively or predominantly breastfed; mixed feeding was more common in late lactation, increasing from 3 to $23.3 \%$; regardless of breastfeeding practice, frequency per $24 \mathrm{~h}$ averaged 11 feeds per day, which aligns with current recommendations.

\section{Breast Milk Microbiome Community Differs Between Lactation Stages}

Sample extractions yielded significantly different concentrations of total genomic DNA between early and late groups at an average of $11.9 \mathrm{ng} \mu \mathrm{l}( \pm 3.2)$ compared to $3.0 \mathrm{ng} \mu \mathrm{l}( \pm 0.6)$, respectively. A total of 1,505 OTUs were assembled and captured $6,294,781$ sequence reads across all 76 breast milk samples.
These could be annotated as 287 species (71\% of reads), 180 genera and 23 family or higher taxa as well as 1,015 which couldn't be recognized as $99 \%$ similar (in both identity and coverage) to any known taxa (termed TrueUnknowns). Of the 287 OTUs annotated as putative species, the average BLASTn return identity was $99.8 \%$ including 167 perfect hits (100\% identity). At a phyla level, bacteria from Firmicutes, Proteobacteria, Actinobacteria, and Bacteroidetes were most prevalent in comprising $48,44,6$, and $1 \%$ of sequenced

TABLE 1 | Characteristics of Guatemalan mothers at each lactation stage.

\begin{tabular}{|c|c|c|c|}
\hline & \multicolumn{3}{|c|}{ Lactation stages } \\
\hline & $\begin{array}{c}\text { Total mean } \pm \\
\text { SD or } \% \\
(95 \% \mathrm{Cl})\end{array}$ & $\begin{array}{c}\text { Early mean } \pm \\
\text { SD or } \% \\
(95 \% \mathrm{Cl})\end{array}$ & $\begin{array}{c}\text { Late mean } \pm \\
\text { SD or } \% \\
(95 \% \mathrm{Cl})\end{array}$ \\
\hline \multicolumn{4}{|l|}{ Region (\%) } \\
\hline Los Alonzo & 13.1 & 18.2 & 9.3 \\
\hline Buena Vista & 25.0 & 12.1 & 34.9 \\
\hline Espumpujá & 2.6 & 6.1 & 0.0 \\
\hline Los López & 3.9 & 9.1 & 0.0 \\
\hline Los Pérez & 9.2 & 3.0 & 14.0 \\
\hline Los Romero & 27.6 & 36.4 & 20.9 \\
\hline La Unión & 9.2 & 6.1 & 11.6 \\
\hline La Esperanza & 9.2 & 9.1 & 9.3 \\
\hline Maternal age (years) & $23.2 \pm 5.8$ & $22.7 \pm 5.5$ & $23.6 \pm 6.0$ \\
\hline Maternal height (cm) & $146.7 \pm 5.1$ & $146.2 \pm 4.7$ & $147.2 \pm 5.4$ \\
\hline Maternal weight (kg) & $50.8 \pm 7.6$ & $50.2 \pm 6.7$ & $51.2 \pm 8.3$ \\
\hline Maternal BMI $\left(\mathrm{kg} / \mathrm{m}^{2}\right)$ & $23.5 \pm 3.1$ & $23.5 \pm 2.8$ & $23.5 \pm 3.4$ \\
\hline \multicolumn{4}{|l|}{ Parity } \\
\hline Primiparous & 49.0 & 45.0 & 52.0 \\
\hline Multiparous & 51.0 & 55.0 & 48.0 \\
\hline \multicolumn{4}{|l|}{ Marital status (\%) } \\
\hline Married & 25.0 & 27.0 & 22.5 \\
\hline United & 73.0 & 73.0 & 72.5 \\
\hline Single & 3.0 & 0.0 & 5.0 \\
\hline \multicolumn{4}{|l|}{ Education (\%) } \\
\hline No & 81.0 & 85.0 & 76.0 \\
\hline $\begin{array}{l}\text { Primary education or } \\
\text { higher }\end{array}$ & 19.0 & 15.0 & 24.0 \\
\hline \multicolumn{4}{|l|}{$\begin{array}{l}\text { Delivery location and } \\
\text { attendant }\end{array}$} \\
\hline $\begin{array}{l}\text { Informal sector and } \\
\text { midwife }\end{array}$ & 78.0 & 85.0 & 73.0 \\
\hline Hospital and formal & 22.0 & 15.0 & 28.0 \\
\hline \multicolumn{4}{|l|}{$\begin{array}{l}\text { Infant feeding } \\
\text { practices }\end{array}$} \\
\hline Exclusive & 47.4 & 51.5 & 44.2 \\
\hline Predominant & 38.2 & 45.5 & 32.6 \\
\hline Mixed & 14.5 & 3.0 & 23.3 \\
\hline Breastfeeding First hour & 61.0 & 53.0 & 68.0 \\
\hline Frequency/24 h & $11.9 \pm 4.3$ & $11.7 \pm 4.7$ & $12.1 \pm 4$ \\
\hline \multicolumn{4}{|l|}{ Infant first food } \\
\hline Breast & 88.0 & 88.0 & 87.5 \\
\hline Agüitas & 11.0 & 12.0 & 10.0 \\
\hline Beverages & 1.0 & 0.0 & 2.5 \\
\hline
\end{tabular}


amplicons, respectively (Supplementary File 1). The most abundant species across all samples were Streptococcus salivarius and Novosphingobium clariflavum making up 7.54 and $6.22 \%$ amplicons, respectively.

Distance-based ordination using sparsity filtered raw count matrix was performed with five different categorical variables; four were dichotomous (lactation stage, parity, BMI, and mother age) and one (breastfeeding practice) was based on three factors (exclusive, predominant, or mixed). Constrained Analysis of Principal Coordinates (CAP) analysis best segregated mothers based on lactation stage (Figure 1) and identified significant differences with Monte Carlo permutation testing between sample groups (10,000 permutations) at $p<0.001$; parity, $\mathrm{BMI}$, mother age and breastfeeding practice were not significant ( $p>0.1$ in all cases). Shannon and Inverse Simpson indices, used to estimate microbiome diversity, were not significantly ( $t$-test $p<0.05)$ higher in late compared to early lactation stage breast milk (Figure 1).

In contrast, differential abundance analysis using DESeq2 identified taxa which were significantly different in relative abundance between early and late lactation stages. In total, 137 OTUs were identified as significantly differentially abundant, including 39 which were in higher relative abundance in early stage milk and the remaining 98 higher in late stage milk (Figure 2 and Supplementary File 2). These could be annotated at various taxonomic levels, including: 60 species, 25 genera, 1 family as well as 52 which could not be recognized (TrueUnknowns). A general shift in differential abundance was observed with lower relative abundance in some taxa from Firmicutes in early stage milk which were replaced by taxa from Proteobacteria in late stage milk. This was driven by Streptococcus and Staphylococcus species present at higher relative abundance within early milk whereas Sphingomonadaceae and Pseudomonas were higher in late milk, with the majority of differentially abundant species in each respective taxa group being inversely correlated (Figure 3 and Supplementary File 1).

\section{Bacterial Species Associated With Early Lactation}

Ten distinct Staphylococcus OTUs were identified as having significantly higher relative abundance in early milk, including the species: S. hominis, S. epidermidis, and S. hyicus (Figure 2). The most abundant Staphylococcus OTU (Staphylococcus_MS_6) was ambiguous and could represent one or more of the species S. epidermidis, S. haemolyticus, or S. hominis.

Seventeen differentially abundant OTUs with higher abundance in early lactation stage breast milk were identified as putative species within the genus Streptococcus. These included S. mitis, S. parasanguinis, S. peroris, S. pneumoniae, S. pseudopneumoniae, and S. salivarius as well as four OTUs which were ambiguous and could represent a number different of species. Beyond Staphylococcus and Streptococcus, other OTUs representing species identified as significantly higher in relative abundance in early milk included: Corynebacterium tuberculostearicum, Corynebacterium jeikeiu, Lactobacillus gasseri, Acinetobacter johnsonii, Kocuria palustris, and Janthinobacterium agaricidamnosum.

\section{Bacterial Species Associated With Late Lactation}

The general pattern of higher Staphylococcus and specific Streptococcus species in early milk was also reflected by corresponding significant $(p<0.05)$ inverse correlations with Sphingobium yanoikuyae, Pseudomonas putida, Stenotrophomonas maltophilia, Ottowia beijingensis, and Comamonas testosteroni, all of which were significantly differentially abundant with higher relative abundance in late breast milk.

Five distinct $S$. yanoikuyae OTUs and a S. limneticum OTU were identified as significantly higher in late milk. The two most abundant OTUs, Sphingobium yanoikuyae_4 and Sphingobium yanoikuyae_5, likely represent distinct strains in sharing 100\% identity with Sphingobium yanoikuyae S72 and ATCC 51230, respectively, with each strain harboring four identical gene copies. Additionally, a single Novosphingobium clariflavum OTU was identified as differentially abundant and higher in late breast milk. The Novosphingobium clariflavum OTU was particularly high in abundance in the majority of late breast milk samples and was the most abundant OTU in $51 \%$ of late milk samples, representing an average of $25 \%$ of raw reads.

Nine Pseudomonas OTUs were differentially expressed with eight having higher relative abundance in late milk. These included four $P$. putida and one $P$. fulva OTU as well as three ambiguous OTUs which could be P. putida, P. fulva, $P$. parafulva, and/or P. gingeri. Stenotrophomonas maltophilia (closely related to Pseudomonas), Cloacibacterium normanense, Flavobacterium cucumis, Ottowia beijingensis (absent from early milk samples), Prevotella melaninogenica, Veillonella dispar, Actinomyces odontolyticus as well as the highly abundant Comamonas testosteroni, were also each identified as significantly more abundant in late lactation stage breast milk.

A number of poorly characterized OTUs were also identified as having higher relative abundance in late milk. These were dominated by unknown sequences previously observed in samples from oral or saliva microbiome studies, such as: TrueUnknown_ 944 and TrueUnknown_ 978 (97.5\% similar Streptococcus sanguinis SK1), TrueUnknown_967 and TrueUnknown_980 (98\% similar Prevotella sp.), TrueUnknown_915 (98\% similar Granulicatella elegans), as well as the high relative abundance TrueUnknown_926 which was $97 \%$ similar to a (challenging to culture) TM7 group bacterium often found in oral samples.

\section{DISCUSSION}

\section{General Characteristics}

Breast milk supplies a continuous source of bacteria during lactation (Fitzstevens et al., 2017) and there is consensus that this provision inoculates the gastrointestinal tract with commensal or beneficial bacteria (Martín et al., 2004; Ramsay et al., 2004; Moossavi et al., 2019; Williams et al., 2019). However, 

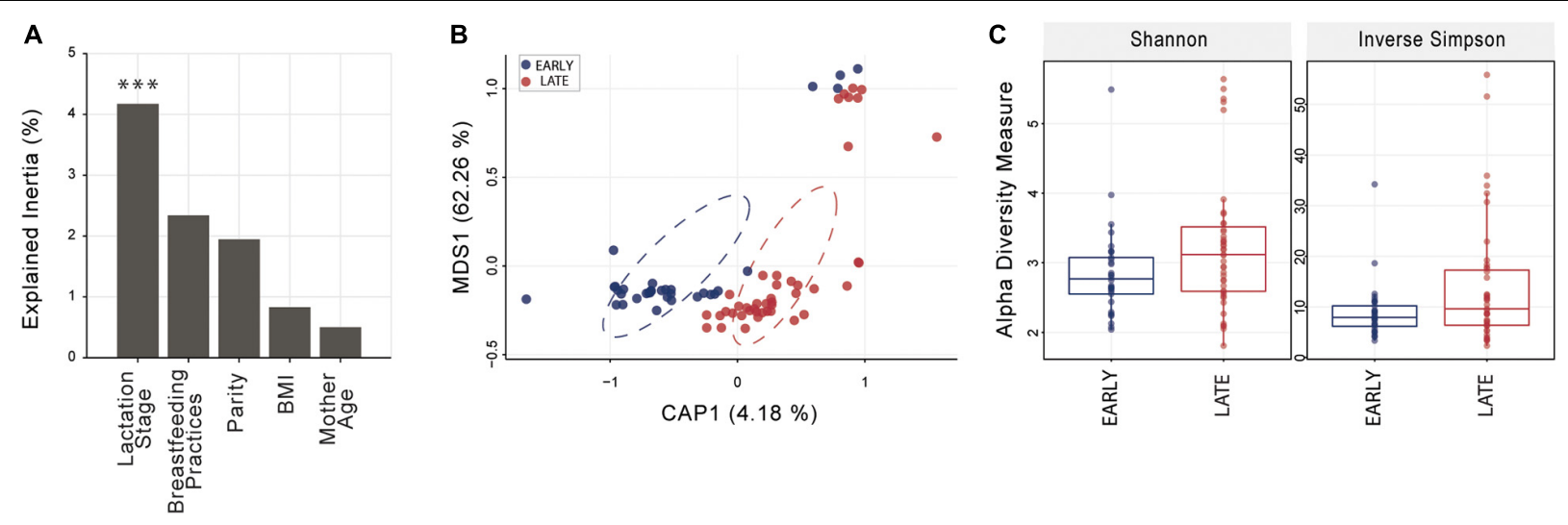

FIGURE 1 | Breast milk community ordination and alpha diversity comparing early and late lactation microbiome. (A) Variation explained by the tested variables in constrained ordination (CAP). Significance testing using Monte Carlo permutation test (10,000 permutation; $\left.{ }^{\star \star \star} p<0.001\right)$. (B) Lactation stage CAP, circles represent sample density relative to each group. (C) Alpha diversity indices were not significantly different (t-test, $p>0.05)$ between early $(n=33)$ and late $(n=43)$ lactation groups. See Supplementary File 1 for an overview of OTU taxonomy.

this milk bacterial community is thought to be influenced by the communities present in other maternal tissues (e.g., skin and the gastrointestinal tract) as well as the infant oral cavity (e.g., "retrograde flow") and the environment (Jost et al., 2014; LaTuga et al., 2014; Rodríguez, 2014). Despite this, the microbiome of breast milk is still poorly understood, and no consensus has been reached on the "core" genera microbiome (Jeurink et al., 2012; Fitzstevens et al., 2017) or if dynamic changes in this bacterial community might occur throughout lactation. In our study, unrelated mothers living in eight remote but distinct communities with little possibility of exchanging microbes among one another were studied. Moreover, this indigenous Guatemalan population was chosen because nearly $100 \%$ exclusively or predominantly breastfed for 6 months (Wren et al., 2015), complying with WHO recommendations to actively breastfeed during this period (World Health Organization, 2014). This is considerably higher than the global average of $41 \%$ of mothers breastfeeding for 6 months, which falls to as low as $26 \%$ in North America (UNICEF, 2018). Importantly, this study population also represented a cohort of mothers who are not from a high-income country, which currently dominates the research field. Thus, this study contributes toward identifying common characteristics and possible biases generated from a shortfall in participant diversity from under-represented communities in breast milk microbiome research.

\section{Early Lactation Stage Breast Milk Is Dominated by Commensal Bacterial Species}

Although previous studies have reported differences in the human milk microbiome related to maternal BMI and stage of lactation (Cabrera-Rubio et al., 2012; Williams et al., 2017, 2019; Moossavi et al., 2019), few have characterized the milk microbiome by identifying changes in the relative abundance of putative species. The benefit of high-resolution microbiome analysis at species level is that, although many species remain poorly characterized, the potential for association to established functions is improved when compared to annotation at phyla, family or genera level. Species-level annotation however, even when sequences share 100\% 16S rRNA gene fragment similarity to a single species, should be considered as putative as substantial mistakes exist in major repositories (Gonzalez et al., 2019). The two most abundant species identified in higher relative abundance in early lactation stage were the coagulase negative Staphylococcus hominis and Staphylococcus epidermidis (Figure 2), both of which are ubiquitous in humans and have been previously associated with opportunistic pathogenicity (Coates et al., 2014). The higher abundance of S. hominis and S. epidermidis in early milk is consistent with findings that nonvirulent strains of these species are likely early gut pioneers of term infants, and may have the potential to reduce colonization by more virulent species (Soeorg et al., 2017). In our study, it was also interesting to note that although both species are recognized as common skin bacteria and common inhabitants of healthy breast milk environment (Heikkilä and Saris, 2003; Martín et al., 2007), their relative abundance was lower in late milk.

Streptococcus mitis and Streptococcus salivarius strains are usually considered commensal, are commonly isolated from milk (Kilian et al., 2014; Delorme et al., 2015; Sørensen et al., 2016) and have been identified as infant oral microbiome inhabitants within the first days after birth (Pearce et al., 1995). The OTUs from both species were generally in higher relative abundance in early milk (Figure 2). An exception to this was in S. mitis, where two of the six differentially abundant $S$. mitis OTUs (Streptococcus_mitis_9 and Streptococcus_mitis_12) had higher relative abundance in late milk. Interestingly, the most abundant $S$. mitis OTU in early (Streptococcus_mitis_14) and late (Streptococcus_mitis_9) lactation stages share $100 \%$ identity to distinct strains, Strep mitis RH_12363_08 and Strep mitis SK637, respectively. These OTUs, alongside the others identified as significantly varying between early and late lactation stages are most similar to different S. mitis phylogenetic sub-clades (Supplementary File 1; NCBI Genome Tree report), in line with previously described strain 


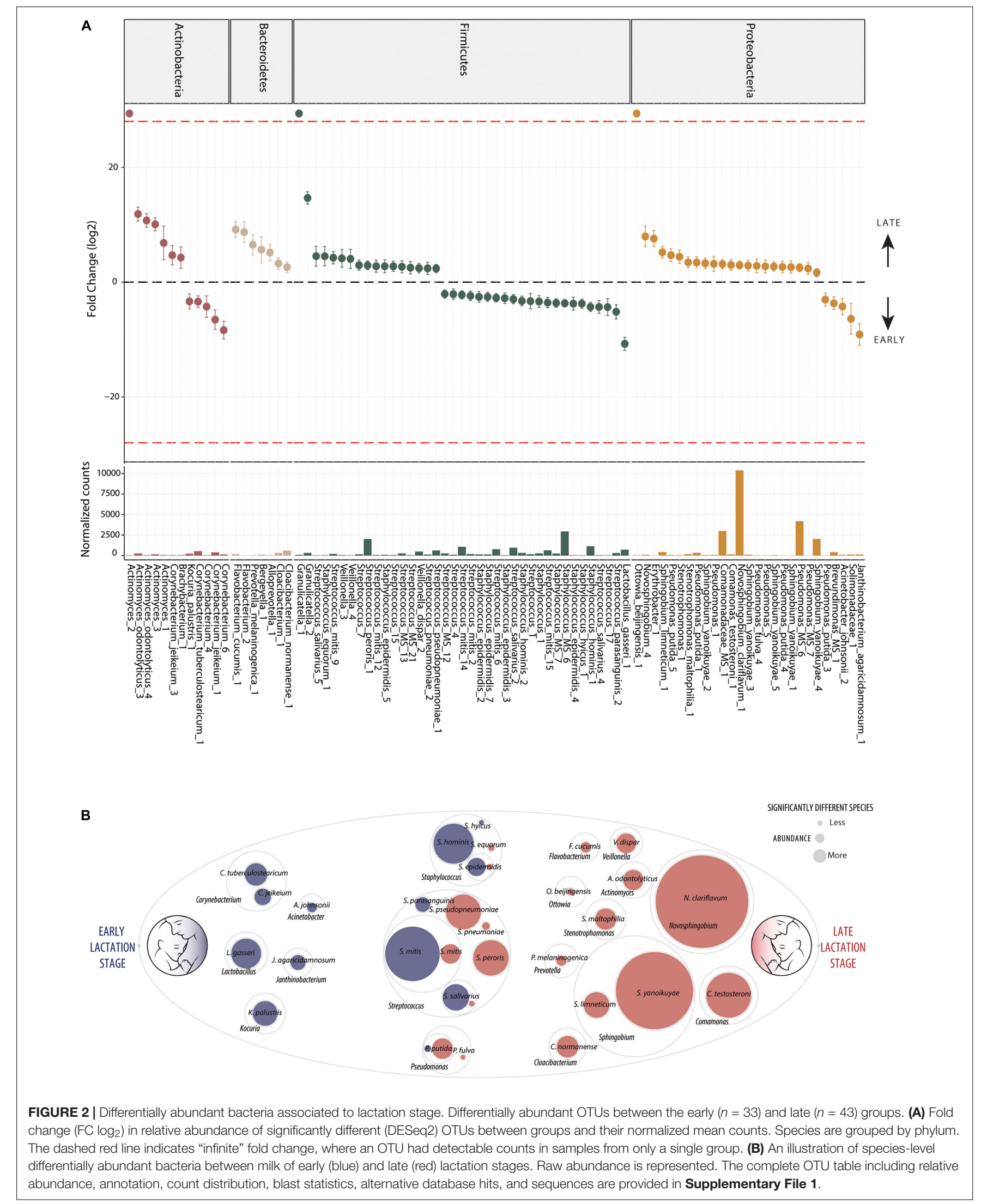




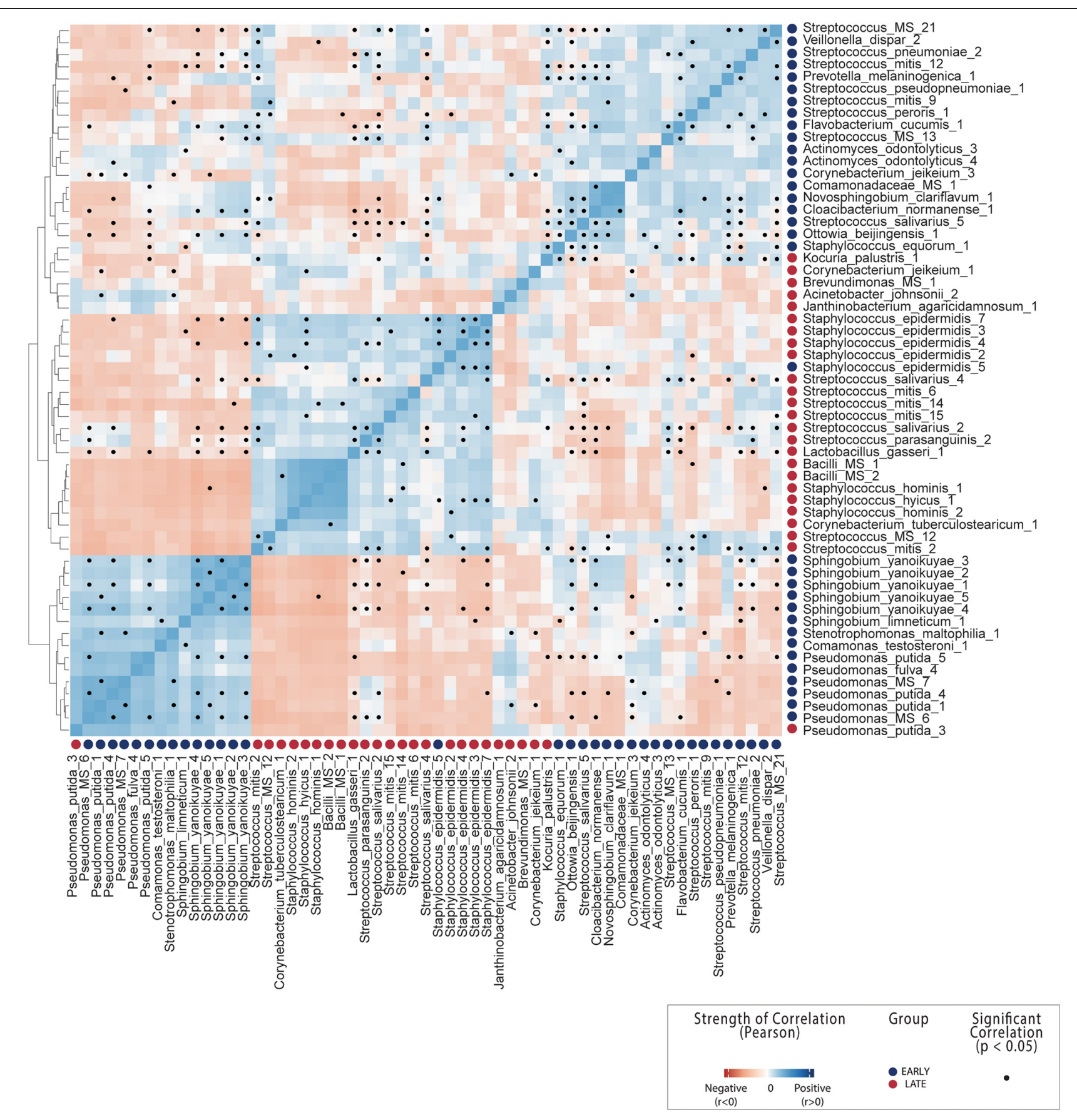

FIGURE 3 | OTU-OTU correlations across all breast milk samples. Count correlation of all OTUs differentially abundant between early and late lactation stages which could be annotated at species level. Colors indicate positive (blue) or negative (red) correlation and black dots indicate significance $(p<0.05)$. See Supplementary File 1 correlations including non-differentially abundant of OTUs from mothers.

phylogeny (Rasmussen et al., 2016). Although little is known about functional variation between these strains, these results suggest that such variation does exist.

Species from the genus Corynebacterium are very commonly isolated from human breast milk and are generally considered commensal in nature (Hunt et al., 2011; McGuire and McGuire, 2017). The two species identified as higher in relative abundance in early milk could be a concern as both C. tuberculostearicum (which was first identified as leprosy-derived Corynebacterium and C. jeikeium) have historically been considered pathogenic (Riegel et al., 1994; Paviour et al., 2002; Hinić et al., 2012), although recent evidence has indicated that these species can be commensal (Damaceno et al., 2017). Similarly, Acinetobacter johnsonii, which has been previously, although not commonly, 
detected in breast milk and is known to utilize lactate (Bouvet and Grimont, 1986; Delgado et al., 2008), could be a potential health concern as it has been associated with mastitis (Patel et al., 2017). Kocuria palustris, first isolated from rhizosphere samples (Kovács et al., 1999), was also unexpected within early breast milk as the species has not previously been reported in breast milk samples; although the genera has been detected in both ruminant and human milk (Jeurink et al., 2012; Li et al., 2017; Lackey et al., 2019).

A number of species characterized as potentially beneficial to infant health were also identified as significantly enriched in early breast milk. Lactobacillus gasseri is well characterized as a species with some strains that produce bacteriocins (gassericin A) (Pandey et al., 2013; Mousa et al., 2017) and is widely considered a putative early colonizer of infant guts. It is subsequently heavily marketed as a probiotic due to the potential to provide host resistance to infections from pathogens such as Listeria monocytogenes (Kawai et al., 2004; Selle and Klaenhammer, 2013), although evidence of this function in situ has not been yet been generated. L. gasseri was significantly positively correlated with S. salivarius and $S$. parasanguinis here (Figure 3 and Supplementary File 1) and inversely correlated with the Sphingobium and Pseudomonas species in late milk microbiome. Another species with potential antimicrobial properties in significantly higher relative abundance in early lactation stage breast milk was Janthinobacterium agaricidamnosum, which produces an antifungal compound (jagaricin) with strong antifungal activity against major human fungal pathogens (Lincoln et al., 1999; Graupner et al., 2012, 2015). Although the species could potentially improve infant biotic resistance, it has not previously been observed in milk and is largely uncharacterized (jagaricin also has hemolytic activity; Fischer et al., 2019).

\section{Species Associated With Late Lactation Stage Breast Milk Have a Common Function}

An inverse correlation between bacteria within the phyla Firmicutes and Proteobacteria has recently been observed in human breast milk (Moossavi et al., 2019). The major genera identified here as representing this shift toward Proteobacteria in later lactation (Figure 2), Sphingobium and Novosphingobium, are often found in human breast milk and have been previously identified as highly abundant together with Pseudomonas in milk of healthy mothers in a non-marker based metagenomic study (Jiménez et al., 2015). Sphingobium yanoikuyae, identified here as significantly higher in relative abundance in late breast milk, is a well-known soil bioremediation species with some strains having polycyclic aromatic hydrocarbons (PAH) degradation activity in the environment (Cunliffe and Kertesz, 2006; Kou et al., 2018) but was first isolated from human clinical samples (Yabuuchi et al., 1990). Recruitment of bacteria with such functionality could be a response to accumulation of xenobiotics such as PAHs and polychlorinated biphenyls, which can accumulate in fatty tissue and breast milk (Dekoning and Karmaus, 2000), although this would not explain association with lactation stage.
On the other hand, this species has also been previously observed as associated with nipple aspirate fluid of healthy women in a breast cancer study, where the authors speculated that the $\mathrm{PAH}$ degrading activity may provide protection against cancer tumor development (Chan et al., 2016; Parida and Sharma, 2019). Finally, although $N$. clariflavum was very widely distributed throughout samples, the species has yet to be characterized in detail (Zhang et al., 2017) and has never been previously identified in breast milk samples. However, Novosphingobium have been isolated from a very wide range of habitats including soils, plants and water, and are known as aromatic hydrocarbon compound degraders expected within soils and rhizospheres contaminated with persistent organic pollutants (Kumar et al., 2017; Wang et al., 2018).

The pattern of hydrocarbon degradation functionality was consistent with the majority of species identified as significantly enriched in late breast milk (Figure 2). Pseudomonas putida strains are common soil and rhizosphere degraders of persistent organic pollutants which are used for bioremediation as it can metabolize aromatic hydrocarbons as a sole carbon source (Feist and Hegeman, 1969; Dunn and Gunsalus, 1973; Marqués and Ramos, 1993), including caffeine (Summers et al., 2015). While Pseudomonas species are commonly observed in metagenomic studies of healthy mothers (Jeurink et al., 2012; Fitzstevens et al., 2017), they are generally considered environmental contamination in human breast milk (Jiménez et al., 2015). The closely related Stenotrophomonas maltophilia, which is considered non-lactose fermenting, has previously been identified as an opportunistic pathogen associated with cystic fibrosis, where it can form mixed species biofilm with Pseudomonas aeruginosa (Esposito et al., 2017). Given an established capability to cooperatively associate with $P$. aeruginosa, it is interesting to note that the species was significantly correlated in our study with P. putida (Figure 3).

Consistent with the differential abundance of Sphingobium yanoikuyae and $P$. putida, strains of Stenotrophomonas maltophilia also have extensive hydrocarbon degradation activity (Singh and Fulekar, 2010; Arulazhagan et al., 2017). The same is true of Cloacibacterium normanense, which is often isolated from wastewater and can often grow on oily sludge as its sole carbon source (Cerqueira et al., 2012), and Comamonas testosteroni, which has been isolated from cow milk (Quigley et al., 2013) and has strains associated with (eponymously) steroid and broader aromatic hydrocarbon degrading (Kamimura et al., 2010; Horinouchi et al., 2018). Ottowia beijingensis, first isolated from phenol degrading consortia in wastewater sludge, and Flavobacterium cucumis have not previously been reported in breast milk, to the authors' knowledge, but both have strains with hydrocarbon degradation capability (Al-Mailem et al., 2014; Cao et al., 2014; Fernandez-Gonzalez et al., 2018), consistent with the majority of differentially abundant species significantly enriched in late milk.

Other species significantly enriched in late milk have previously been isolated from an expected common sourcethe oral cavity, including Prevotella melaninogenica, Veillonella dispar and Actinomyces odontolyticus. Actinomyces odontolyticus is often considered pathogenic and strains have been associated 
with infection/mastitis (Hoyles et al., 2001; Bing et al., 2015), but it is also a common oral commensal species which has been observed to increase during puberty (Gusberti et al., 1990). $P$. melaninogenica is a common oral commensal bacteria which has been suggested as transmitted directly from mother to colonize the infant mouth via saliva (Könönen et al., 1994), and has also been suggested for the oral commensal Granulicatella (Dewhirst et al., 2010; Gomez and Nelson, 2017). Species such as $V$. dispar, identified here as significantly higher in relative abundance in late milk, has been recently discussed with relevance to infant age (being commonly isolated from the oral cavity) (Tuominen et al., 2018) and strains have been identified in late (3 months postpartum) milk alongside Granulicatella (Simpson et al., 2018) and uncharacterized species from the hard to culture TM7 group of bacteria Candidatus saccharibacteria (Cabrera-Rubio et al., 2012). These findings suggest important roles of poorly characterized and unknown bacteria associated to lactation stage, highlighting that a substantial amount is yet to be discovered about breast milk as an ecosystem.

\section{Limitations}

Differentially abundant species are presented here based on amplified 16S rRNA gene sequences alone and do not establish viability of bacteria putatively identified. Future research confirming these findings should attempt to isolate species identified using culturing techniques. A concern often raised in milk microbiome studies relates to primer selection, an important limitation in bacterial marker technology. Inconsistency in the perceived "core" genera of bacteria commonly identified in the breast milk of healthy mothers is well captured in the systematic human breast milk microbiome reviews of Jeurink et al. (2012) and Fitzstevens et al. (2017) where most of the major studies report Staphylococcus, Streptococcus, Pseudomonas and either Bifidobacterium or Cutibacterium (formally Propionibacterium). This observed inconsistency between Bifidobacterium and Cutibacterium originates from the fundamental limitation of bacterial marker technology that there are no universal primers for the 16S rRNA gene (Klindworth et al., 2013; Gonzalez et al., 2019). Sequence analysis has revealed that the difference between these studies relates to a bias associated with primer selection. Specifically, the primers 27F/533R (utilized in this study), targets the V1-V3 region of the $16 \mathrm{~S}$ rRNA gene and are often used in breast milk microbiome studies (CabreraRubio et al., 2012; Mediano et al., 2017; Lackey et al., 2019) can successfully amplify the putative "core" breast milk genus Cutibacterium (formally Propionibacterium) (Hunt et al., 2011; Jost et al., 2013; Jiménez et al., 2015) but cannot amplify species from the putative "core" genus Bifidobacterium (Klindworth et al., 2013). Conversely, the other most commonly used primers, the $515 \mathrm{~F} / 806 \mathrm{R}$ primer pair (V4 region targeting), do amplify species within the genus Bifidobacterium but do not amplify species from the genera Cutibacterium (Klindworth et al., 2013), although Bifidobacterium sp. are often (Moossavi et al., 2019) but not always (Davé et al., 2016; Ramani et al., 2018) identified in breast milk studies when such primers are used. Whole genome shotgun (WGS) approaches can serve to resolve these types of identification bias generated from
$16 S$ rRNA primer selection; however, quantitative differential abundance analysis using WGS approaches remains a technical challenge for studies involving high sample numbers and with high bacterial diversity, such as is present within breast milk. Although Pseudomonas sp. are normally identified in breast milk of healthy mothers (Martín et al., 2007; Hunt et al., 2011, 2012; Jost et al., 2013; Ward et al., 2013; Jiménez et al., 2015; Urbaniak et al., 2016; McGuire and McGuire, 2017; Moossavi et al., 2019) species from the genera are common in the environment and could represent contamination (Jiménez et al., 2015; Treven et al., 2019), particularly as late breast milk samples had lower average DNA concentrations, although recent evidence suggests Pseudomonas presence in breast milk is genuine (Douglas et al., 2020).

\section{CONCLUSION}

Evidence is provided of extensive remodeling of the breast milk microbiome across lactation stages in a cohort of healthy unrelated Guatemalan mothers. These include a microbiome shift from specific Staphylococcus and Streptococcus species at the beginning of lactation toward Sphingobium and Pseudomonas species at a later stage of lactation. Collectively, the findings reveal that common changes occur in the healthy human breast milk microbiome over the first 6 months of lactation and highlight the importance of investigating the microbiome at different stages of lactation. The large majority of species significantly associated with later lactation, such as Sphingomonas yanoikuyae, Pseudomonas putida, Stenotrophomonas maltophilia, Cloacibacterium normanense, Comamonas testosteroni, Ottowia beijingensis, and Flavobacterium cucumis are intriguing in having the common functional trait of aromatic hydrocarbon degradation. Whilst the contribution of specific bacterial species to infant metabolism and immunity is largely opaque, these distinct changes suggest active on-going remodeling of the milk microbiome throughout lactation that could contribute favorably to infant health. Moreover, these insights illustrate the need to study under-represented human communities in breast milk research given the complexity of cross-talk between the mother-infant dyad during lactation.

\section{DATA AVAILABILITY STATEMENT}

All data are made available within the manuscript or Supplementary Data. Raw sequence data has been deposited at the European Genome-Phenome Archive (EGAD00001004160) and are available upon request to KK, kristine.koski@mcgill.ca.

\section{ETHICS STATEMENT}

The studies involving human participants were reviewed and approved by McGill Institutional Review Board and CeSSIAM 
Human Subjects Committee. All participating mothers provided written informed consent for participation.

\section{AUTHOR CONTRIBUTIONS}

EG and NB analyzed microbiome data, created figures, and drafted the manuscript. CL drafted the manuscript, prepared samples for the $16 \mathrm{~S}$ rRNA sequencing and LL prepared data files of maternal characteristics. NS supervised milk sample collection. KK, CL, LA, and MS conceptualized the study design and edited the final manuscript. NS, LA, and KK provided the financial support. All authors contributed to the article and approved the submitted version.

\section{FUNDING}

This study was supported by the Natural Sciences and Engineering Research Council of Canada (NSERC): NSERC RGPIN-2015-04390 and NSERC RGPIN-2016-04961.

\section{REFERENCES}

Al-Mailem, D., Kansour, M., and Radwan, S. (2014). Bioremediation of hydrocarbons contaminating sewage effluent using man-made biofilms: effects of some variables. Appl. Biochem. Biotechnol. 174, 1736-1751. doi: 10.1007/ s12010-014-1067-z

Anders, S., McCarthy, D. J., Chen, Y., Okoniewski, M., Smyth, G. K., Huber, W., et al. (2013). Count-based differential expression analysis of RNA sequencing data using R and Bioconductor. Nat. Protoc. 8:1765. doi: 10.1038/nprot.2013. 099

Andreas, N. J., Kampmann, B., and Le-Doare, K. M. (2015). Human breast milk: A review on its composition and bioactivity. Early Hum. Dev. 91, 629-635. doi: 10.1016/j.earlhumdev.2015.08.013

Arulazhagan, P., Al-Shekri, K., Huda, Q., Godon, J.-J., Basahi, J. M., and Jeyakumar, D. (2017). Biodegradation of polycyclic aromatic hydrocarbons by an acidophilic Stenotrophomonas maltophilia strain AJH1 isolated from a mineral mining site in Saudi Arabia. Extremophiles 21, 163-174. doi: 10.1007/ s00792-016-0892-0

Biagi, E., Quercia, S., Aceti, A., Beghetti, I., Rampelli, S., Turroni, S., et al. (2017). The bacterial ecosystem of mother's milk and infant's mouth and gut. Front. Microbiol. 8:1214. doi: 10.3389/fmicb.2017.01214

Bing, A., Loh, S., Morris, T., Hughes, H., Dixon, J., and Helgason, K. (2015). Actinomyces species isolated from breast infections. J. Clin. Microbiol. 53, 3247-3255. doi: 10.1128/jcm.01030- 15

Bolyen, E., Rideout, J. R., Dillon, M. R., Bokulich, N. A., Abnet, C. C., AlGhalith, G. A., et al. (2019). Reproducible, interactive, scalable and extensible microbiome data science using QIIME 2. Nat. Biotechnol. 37, 852-857.

Bouvet, P. J., and Grimont, P. A. (1986). Taxonomy of the genus Acinetobacter with the recognition of Acinetobacter baumannii sp. nov., Acinetobacter haemolyticus sp. nov., Acinetobacter johnsonii sp. nov., and Acinetobacter junii sp. nov. and emended descriptions of Acinetobacter calcoaceticus and Acinetobacter lwoffii. Int. J. Syst. Evol. Microbiol. 36, 228-240. doi: 10.1099/ 00207713-36-2-228

Brereton, N., Gonzalez, E., Desjardins, D., Labrecque, M., and Pitre, F. (2019). Co-cropping with three phytoremediation crops influences rhizosphere microbiome community in contaminated soil. Sci. Total Environ. 711:135067. doi: 10.1016/j.scitotenv.2019.135067

Cabrera-Rubio, R., Collado, M. C., Laitinen, K., Salminen, S., Isolauri, E., and Mira, A. (2012). The human milk microbiome changes over lactation and is

\section{ACKNOWLEDGMENTS}

Special thanks to McGill University and Genome Quebec Innovation Centre for technical assistance with 16S rRNA sequencing. We thank $\mathrm{H}$. Wren, AM Chomat, and CeSSIAM field team for sample collection and the participating mothers for providing valuable milk samples.

\section{SUPPLEMENTARY MATERIAL}

The Supplementary Material for this article can be found online at: https://www.frontiersin.org/articles/10.3389/fmicb. 2021.557180/full\#supplementary-material

Supplementary File 1 | Total amplified community flower diagram illustrating raw count distribution across all OTUs.

- Count correlation of 200 OTUs including all mothers.

- Streptococcus mitis strain genome clustering. Streptococcus mitis DA is strain cluster. Strain level research has been conducted (Jensen et al., 2016) but this tree is based on all the up-to-date genomes submitted to NCBI.

Supplementary File 2 | OTU table and lactation DA table.

shaped by maternal weight and mode of delivery. Am. J. Clin. Nutr. 96, 544-551. doi: 10.3945/ajen.112.037382

Cao, J., Lai, Q., Liu, Y., Li, G., and Shao, Z. (2014). Ottowia beijingensis sp. nov., isolated from coking wastewater activated sludge, and emended description of the genus Ottowia. Int. J. Syst. Evol. Microbiol. 64, 963-967. doi: 10.1099/ijs.0. 054015-0

Cerqueira, V. S., Hollenbach, E. B., Maboni, F., Camargo, F. A., Maria, do Carmo, R. P., et al. (2012). Bioprospection and selection of bacteria isolated from environments contaminated with petrochemical residues for application in bioremediation. World J. Microbiol. Biotechnol. 28, 1203-1222. doi: 10.1007/ s11274-011-0923-z

Chan, A. A., Bashir, M., Rivas, M. N., Duvall, K., Sieling, P. A., Pieber, T. R., et al. (2016). Characterization of the microbiome of nipple aspirate fluid of breast cancer survivors. Sci. Rep. 6:28061.

Chomat, A. M., Solomons, N. W., Koski, K. G., Wren, H. M., Vossenaar, M., and Scott, M. E. (2015). Quantitative methodologies reveal a diversity of nutrition, infection/illness, and psychosocial stressors during pregnancy and lactation in rural Mam-Mayan mother-infant dyads from the Western Highlands of Guatemala. Food Nutr. Bull. 36, 415-440. doi: 10.1177/0379572115610944

Coates, R., Moran, J., and Horsburgh, M. J. (2014). Staphylococci: colonizers and pathogens of human skin. Fut. Microbiol. 9, 75-91. doi: 10.2217/fmb.13.145

Cunliffe, M., and Kertesz, M. A. (2006). Effect of Sphingobium yanoikuyae B1 inoculation on bacterial community dynamics and polycyclic aromatic hydrocarbon degradation in aged and freshly PAH-contaminated soils. Environ. Poll. 144, 228-237. doi: 10.1016/j.envpol.2005.12.026

Cunningham, A. S., Jelliffe, D. B., and Jelliffe, E. P. (1991). Breast-feeding and health in the 1980s: a global epidemiologic review. J. pediatr. 118, 659-666. doi: 10.1016/s0022-3476(05)80023-x

Damaceno, Q. S., Souza, J. P., Nicoli, J. R., Paula, R. L., Assis, G. B., Figueiredo, H. C., et al. (2017). Evaluation of potential probiotics isolated from human milk and colostrum. Probiot. Antimicrob. Prot. 9, 371-379. doi: 10.1007/s12602-0179270-1

Davé, V., Street, K., Francis, S., Bradman, A., Riley, L., Eskenazi, B., et al. (2016). Bacterial microbiome of breast milk and child saliva from lowincome Mexican-American women and children. Pediatr. Res. 79:846. doi: 10.1038/pr.2016.9

Dekoning, E. P., and Karmaus, W. (2000). PCB exposure in utero and via breast milk. A review. J. Expos. Sci. Environ. Epidemiol. 10:285. doi: 10.1038/sj.jea. 7500090 
Delgado, S., Arroyo, R., Martín, R., and Rodríguez, J. M. (2008). PCR-DGGE assessment of the bacterial diversity of breast milk in women with lactational infectious mastitis. BMC Infect. Dis. 8:51. doi: 10.1186/1471-2334-8-51

Delorme, C., Abraham, A.-L., Renault, P., and Guédon, E. (2015). Genomics of Streptococcus salivarius, a major human commensal. Infect. Genet. Evol. 33, 381-392. doi: 10.1016/j.meegid.2014.10.001

Dewhirst, F. E., Chen, T., Izard, J., Paster, B. J., Tanner, A. C., Yu, W.-H., et al. (2010). The human oral microbiome. J. Bacteriol. 192, 5002-5017.

Douglas, C. A., Ivey, K. L., Papanicolas, L. E., Best, K. P., Muhlhausler, B. S., and Rogers, G. B. (2020). DnA extraction approaches substantially influence the assessment of the human breast milk microbiome. Sci. Rep. 10, 1-10.

Dunn, N., and Gunsalus, I. (1973). Transmissible plasmid coding early enzymes of naphthalene oxidation in Pseudomonas putida. J. Bacteriol. 114, 974-979. doi: 10.1128/jb.114.3.974-979.1973

Esposito, A., Pompilio, A., Bettua, C., Crocetta, V., Giacobazzi, E., Fiscarelli, E., et al. (2017). Evolution of Stenotrophomonas maltophilia in cystic fibrosis lung over chronic infection: a genomic and phenotypic population study. Front. Microbiol. 8:1590. doi: 10.3389/fmicb.2017.01590

Feist, C. F., and Hegeman, G. (1969). Phenol and benzoate metabolism by Pseudomonas putida: regulation of tangential pathways. J. Bacteriol. 100, 869877. doi: 10.1128/jb.100.2.869-877.1969

Fernández, L., Langa, S., Martín, V., Maldonado, A., Jiménez, E., Martín, R., et al. (2013). The human milk microbiota: origin and potential roles in health and disease. Pharmacol. Res. 69, 1-10. doi: 10.1016/j.phrs.2012.09.001

Fernandez-Gonzalez, N., Sierra-Alvarez, R., Field, J. A., Amils, R., and Sanz, J. L. (2018). Adaptation of granular sludge microbial communities to nitrate, sulfide, and/or p-cresol removal. Int. Microbiol. 22, 305-316. doi: 10.1007/s10123-01800050-4

Fischer, D., Gessner, G., Fill, T. P., Barnett, R., Tron, K., Dornblut, K., et al. (2019). Disruption of membrane integrity by the bacterium-derived antifungal jagaricin. Antimicrob. Agents Chemother. 63, 00707-00719e.

Fitzstevens, J. L., Smith, K. C., Hagadorn, J. I., Caimano, M. J., Matson, A. P., and Brownell, E. A. (2017). Systematic Review of the Human Milk Microbiota. Nutr. Clin. Pract. 32, 354-364. doi: 10.1177/0884533616670150

Gomez, A., and Nelson, K. E. (2017). The oral microbiome of children: development, disease, and implications beyond oral health. Microbial. Ecol. 73, 492-503. doi: 10.1007/s00248-016-0854-1

Gonzalez, E., Pitre, F., and Brereton, N. (2019). ANCHOR: A 16S rRNA gene amplicon pipeline for microbial analysis of multiple environmental samples. Environ. Microbiol. 21, 2440-2468. doi: 10.1111/1462-2920.14632

Graupner, K., Lackner, G., and Hertweck, C. (2015). Genome sequence of mushroom soft-rot pathogen Janthinobacterium agaricidamnosum. Genome Announc. 3, 277-215e.

Graupner, K., Scherlach, K., Bretschneider, T., Lackner, G., Roth, M., Gross, H., et al. (2012). Imaging mass spectrometry and genome mining reveal highly antifungal virulence factor of mushroom soft rot pathogen. Angewandte Chem. Int. Edition 51, 13173-13177. doi: 10.1002/anie.201206658

Gusberti, F., Mombelli, A., Lang, N., and Minder, C. E. (1990). Changes in subgingival microbiota during puberty: A 4-year longitudinal study. J. Clin. Periodontol. 17, 685-692. doi: 10.1111/j.1600-051x.1990.tb01054.x

Heikkilä, M., and Saris, P. (2003). Inhibition of Staphylococcus aureus by the commensal bacteria of human milk. J. Appl. Microbiol. 95, 471-478. doi: 10. 1046/j.1365-2672.2003.02002.x

Hinić, V., Lang, C., Weisser, M., Straub, C., Frei, R., and Goldenberger, D. (2012). Corynebacterium tuberculostearicum: a potentially misidentified and multiresistant Corynebacterium species isolated from clinical specimens. J. Clin. Microbiol. 50, 2561-2567. doi: 10.1128/jcm.00386-12

Horinouchi, M., Koshino, H., Malon, M., Hirota, H., and Hayashi, T. (2018). Steroid degradation in Comamonas testosteroni TA441: identification of metabolites and the genes involved in the reactions necessary before D-ring cleavage. Appl. Environ. Microbiol. 84, 1324-1318e.

Hoyles, L., Falsen, E., Holmström, G., Persson, A., Sjödén, B., and Collins, M. D. (2001). Actinomyces suimastitidis sp. nov., isolated from pig mastitis. Int. J. Syst. Evolut. Microbiol. 51, 1323-1326. doi: 10.1099/00207713-51-4-1323

Hunt, K. M., Foster, J. A., Forney, L. J., Schütte, U. M. E., Beck, D. L., Abdo, Z., et al. (2011). Characterization of the Diversity and Temporal Stability of Bacterial Communities in Human Milk. PLoS One 6:e21313. doi: 10.1371/journal.pone. 0021313
Hunt, K., Preuss, J., Nissan, C., Davlin, C., Williams, J., Shafii, B., et al. (2012). Human milk oligosaccharides promote the growth of staphylococci. Appl. Environ. Microbiol. 78, 4763-4770. doi: 10.1128/aem.00477-12

Jensen, A., Scholz, C. F., and Kilian, M. (2016). Re-evaluation of the taxonomy of the Mitis group of the genus Streptococcus based on whole genome phylogenetic analyses, and proposed reclassification of Streptococcus dentisani as Streptococcus oralis subsp. dentisani comb. nov., Streptococcus tigurinus as Streptococcus oralis subsp. tigurinus comb. nov., and Streptococcus oligofermentans as a later synonym of Streptococcus cristatus. Int. J. Syst. Evolut. Microbiol. 66, 4803-4820. doi: 10.1099/ijsem.0.001433

Jeurink, P., Van Bergenhenegouwen, J., Jiménez, E., Knippels, L., Fernández, L., Garssen, J., et al. (2012). Human milk: a source of more life than we imagine. Benefic. Microbes 4, 17-30. doi: 10.3920/bm2012.0040

Jiménez, E., de Andrés, J., Manrique, M., Pareja-Tobes, P., Tobes, R., Martínez-Blanch, J. F., et al. (2015). Metagenomic analysis of milk of healthy and mastitis-suffering women. J. Hum. Lact. 31, 406-415. doi: $10.1177 / 0890334415585078$

Jonsson, V., Österlund, T., Nerman, O., and Kristiansson, E. (2016). Statistical evaluation of methods for identification of differentially abundant genes in comparative metagenomics. BMC Genomics 17:78. doi: 10.1186/s12864-0162386-y

Jost, T., Lacroix, C., Braegger, C. P., Rochat, F., and Chassard, C. (2014). Vertical mother-neonate transfer of maternal gut bacteria via breastfeeding. Environ. Microbiol. 16, 2891-2904. doi: 10.1111/1462-2920.12238

Jost, T., Lacroix, C., Braegger, C., and Chassard, C. (2013). Assessment of bacterial diversity in breast milk using culture-dependent and culture-independent approaches. Br. J. Nutrit. 110, 1253-1262. doi: 10.1017/s0007114513000597

Kamimura, N., Aoyama, T., Yoshida, R., Takahashi, K., Kasai, D., Abe, T., et al. (2010). Characterization of the protocatechuate 4, 5-cleavage pathway operon in Comamonas sp. strain E6 and discovery of a novel pathway gene. Appl. Environ. Microbiol. 76, 8093-8101. doi: 10.1128/aem.01863-10

Kawai, Y., Kemperman, R., Kok, J., and Saito, T. (2004). The circular bacteriocins gassericin A and circularin A. Curr. Prot. Peptide Sci. 5, 393-398. doi: 10.2174/ 1389203043379549

Kilian, M., Riley, D. R., Jensen, A., Brüggemann, H., and Tettelin, H. (2014). Parallel evolution of Streptococcus pneumoniae and Streptococcus mitis to pathogenic and mutualistic lifestyles. MBio 5, 1490-1414e.

Klindworth, A., Pruesse, E., Schweer, T., Peplies, J., Quast, C., Horn, M., et al. (2013). Evaluation of general 16S ribosomal RNA gene PCR primers for classical and next-generation sequencing-based diversity studies. Nucleic Acids Res. 41:e1. doi: 10.1093/nar/gks808

Knight, R., Vrbanac, A., Taylor, B. C., Aksenov, A., Callewaert, C., Debelius, J., et al. (2018). Best practices for analysing microbiomes. Nat. Rev. Microbiol. 16, 410-422.

Könönen, E., Saarela, M., Karjalainen, J., Jousimies-Somer, H., Alaluusua, S., and Asikainen, S. (1994). Transmission of oral Prevotella melaninogenica between a mother and her young child. Oral Microbiol. Immunol. 9, 310-314. doi: 10.1111/j.1399-302x.1994.tb00077.x

Kou, S., Vincent, G., Gonzalez, E., Pitre, F. E., Labrecque, M., and Brereton, N. J. (2018). The Response of a 16S Ribosomal RNA Gene Fragment Amplified Community to Lead, Zinc, and Copper Pollution in a Shanghai Field Trial. Front. Microbiol. 9:366. doi: 10.3389/fmicb.2018.00366

Kovács, G., Burghardt, J., Pradella, S., Schumann, P., Stackebrandt, E., and Màrialigeti, K. (1999). Kocuria palustris sp. nov. and Kocuria rhizophila sp. nov., isolated from the rhizoplane of the narrow-leaved cattail (Typha angustifolia). Int. J. Syst. Evol. Microbiol. 49, 167-173. doi: 10.1099/0020771349-1-167

Kumar, R., Verma, H., Haider, S., Bajaj, A., Sood, U., Ponnusamy, K., et al. (2017). Comparative genomic analysis reveals habitat-specific genes and regulatory hubs within the genus Novosphingobium. MSystems 2, 20-17e.

Lackey, K. A., Williams, J. E., Meehan, C. L., Zachek, J. A., Benda, E. D., Price, W. J., et al. (2019). What's Normal? Microbiomes In Human Milk And Infant Feces Are Related To Each Other But Vary Geographically: The INSPIRE Study. Front. Nutrit. 6:45. doi: 10.3389/fnut.2019.00045

LaTuga, M. S., Stuebe, A., and Seed, P. C. (2014). A review of the source and function of microbiota in breast milk. Semin. Reprod. Med. 2, 68-73.

Li, C., Solomons, N. W., Scott, M. E., and Koski, K. G. (2016). Minerals and trace elements in human breast milk are associated with Guatemalan infant 
anthropometric outcomes within the first 6 months. J. Nutrit. 146, 2067-2074. doi: $10.3945 /$ jn.116.232223

Li, Z., Wright, A.-D. G., Yang, Y., Si, H., and Li, G. (2017). Unique bacteria community composition and co-occurrence in the milk of different ruminants. Sci. Rep. 7:40950.

Lincoln, S. P., Fermor, T. R., and Tindall, B. (1999). Janthinobacterium agaricidamnosum sp. nov., a soft rot pathogen of Agaricus bisporus. Int. J. Syst. Evol. Microbiol. 49, 1577-1589. doi: 10.1099/00207713-49-4-1577

Love, M. I., Anders, S., Kim, V., and Huber, W. (2015). RNA-Seq workflow: genelevel exploratory analysis and differential expression. F1000Research 4:1070. doi: 10.12688/f1000research.7035.2

Love, M. I., Huber, W., and Anders, S. (2014a). Moderated estimation of fold change and dispersion for RNA-seq data with DESeq2. Genome Biol. 15:550.

Love, M., Anders, S., and Huber, W. (2014b). Differential analysis of count data-the DESeq2 package. Genome Biol. 15:550.

Marqués, S., and Ramos, J. L. (1993). Transcriptional control of the Pseudomonas putida TOL plasmid catabolic pathways. Mol. Microbiol. 9, 923-929. doi: 10. 1111/j.1365-2958.1993.tb01222.x

Martín, R. O., Langa, S., Reviriego, C., Jiménez, E., Marín, M. A. L., Olivares, M., et al. (2004). The commensal microflora of human milk: new perspectives for food bacteriotherapy and probiotics. Trends Food Sci. Technol. 15, 121-127. doi: 10.1016/j.tifs.2003.09.010

Martín, R., Heilig, H. G., Zoetendal, E. G., Jiménez, E., Fernández, L., Smidt, H., et al. (2007). Cultivation-independent assessment of the bacterial diversity of breast milk among healthy women. Res. Microbiol. 158, 31-37. doi: 10.1016/j. resmic.2006.11.004

McGuire, M. K., and McGuire, M. A. (2017). Got bacteria? The astounding, yet notso-surprising, microbiome of human milk. Curr. Opin. Biotechnol. 44, 63-68. doi: 10.1016/j.copbio.2016.11.013

McMurdie, P. J., and Holmes, S. (2013). phyloseq: an R package for reproducible interactive analysis and graphics of microbiome census data. PLoS One 8:e61217. doi: 10.1371/journal.pone.0061217

Mediano, P., Fernández, L., Jiménez, E., Arroyo, R., Espinosa-Martos, I., Rodríguez, J. M., et al. (2017). Microbial diversity in milk of women with mastitis: potential role of coagulase-negative staphylococci, viridans group streptococci, and corynebacteria. J. Hum. Lactat. 33, 309-318. doi: 10.1177/ 0890334417692968

Minerbi, A., Gonzalez, E., Brereton, N. J., Anjarkouchian, A., Dewar, K., Fitzcharles, M.-A., et al. (2019). Altered microbiome composition in individuals with fibromyalgia. Pain 160, 2589-2602. doi: $10.1097 /$ j.pain. 0000000000001640

Moossavi, S., Sepehri, S., Robertson, B., Bode, L., Goruk, S., Field, C. J., et al. (2019). Composition and variation of the human milk microbiota are influenced by maternal and early-life factors. Cell Host Microbe 25, 324-335 doi: 10.1016/j. chom.2019.01.011

Mousa, W. K., Athar, B., Merwin, N. J., and Magarvey, N. A. (2017). Antibiotics and specialized metabolites from the human microbiota. Nat. Prod. Rep. 34, 1302-1331. doi: 10.1039/c7np00021a

Oksanen, J., Blanchet, F. G., Kindt, R., Legendre, P., Minchin, P. R., O’hara, R., et al. (2017). Package 'vegan' in Community ecology package, version. Vienna: R Core Team.

Pandey, N., Malik, R., Kaushik, J., and Singroha, G. (2013). Gassericin A: a circular bacteriocin produced by lactic acid bacteria Lactobacillus gasseri. World J. Microbiol. Biotechnol. 29, 1977-1987. doi: 10.1007/s11274-013-1368-3

Parida, S., and Sharma, D. (2019). The power of small changes: comprehensive analyses of microbial dysbiosis in breast cancer. Biochim. Biophys. Acta Rev. Cancer 1871, 392-405. doi: 10.1016/j.bbcan.2019.04.001

Patel, S. H., Vaidya, Y. H., Patel, R. J., Pandit, R. J., Joshi, C. G., and Kunjadiya, A. P. (2017). Culture independent assessment of human milk microbial community in lactational mastitis. Sci. Rep. 7:7804.

Paviour, S., Musaad, S., Roberts, S., Taylor, G., Taylor, S., Shore, K., et al. (2002). Corynebacterium species isolated from patients with mastitis. Clin. Infect. Dis. 35, 1434-1440.

Pearce, C., Bowden, G., Evans, M., Fitzsimmons, S., Johnson, J., Sheridan, M., et al. (1995). Identification of pioneer viridans streptococci in the oral cavity of human neonates. J. Med. Microbiol. 42, 67-72. doi: 10.1099/00222615-42-1-67
Quigley, L., O'sullivan, O., Stanton, C., Beresford, T. P., Ross, R. P., Fitzgerald, G. F., et al. (2013). The complex microbiota of raw milk. FEMS Microbiol. Rev. 37, 664-698. doi: 10.1111/1574-6976.12030

Ramani, S., Stewart, C. J., Laucirica, D. R., Ajami, N. J., Robertson, B., Autran, C. A., et al. (2018). Human milk oligosaccharides, milk microbiome and infant gut microbiome modulate neonatal rotavirus infection. Nat. Commun. 9:5010.

Ramsay, D. T., Kent, J. C., Owens, R. A., and Hartmann, P. E. (2004). Ultrasound imaging of milk ejection in the breast of lactating women. Pediatrics 113, 361-367. doi: 10.1542/peds.113.2.361

Rasmussen, L., Dargis, R., Højholt, K., Christensen, J., Skovgaard, O., Justesen, U., et al. (2016). Whole genome sequencing as a tool for phylogenetic analysis of clinical strains of Mitis group streptococci. Eur. J. Clin. Microbiol. Infect. Dis. 35, 1615-1625. doi: 10.1007/s10096-016-2700-2

Riegel, P., De Briel, D., Prévost, G., Jehl, F., and Monteil, H. (1994). Genomic diversity among Corynebacterium jeikeium strains and comparison with biochemical characteristics and antimicrobial susceptibilities. J. Clin. Microbiol. 32, 1860-1865. doi: 10.1128/jcm.32.8.1860-1865.1994

Rodríguez, J. M. (2014). The origin of human milk bacteria: is there a bacterial entero-mammary pathway during late pregnancy and lactation? Adv. Nutr. 5, 779-784. doi: 10.3945/an.114.007229

Ruiz, L., García-Carral, C., and Rodriguez, J. M. (2019). Unfolding the human milk microbiome landscape in the omics era. Front. Microbiol. 10:1378. doi: $10.3389 /$ fmicb. 2019.01378

Sakwinska, O., and Bosco, N. (2019). Host microbe interactions in the lactating mammary gland. Front. Microbiol. 10:1863. doi: 10.3389/fmicb.2019.01863

Schloss, P. D., Westcott, S. L., Ryabin, T., Hall, J. R., Hartmann, M., Hollister, E. B., et al. (2009). Introducing mothur: open-source, platform-independent, community-supported software for describing and comparing microbial communities. Appl. Environ. Microbiol. 75, 7537-7541. doi: 10.1128/aem. 01541-09

Selle, K., and Klaenhammer, T. R. (2013). Genomic and phenotypic evidence for probiotic influences of Lactobacillus gasseri on human health. FEMS Microbiol. Rev. 37, 915-935. doi: 10.1111/1574-6976.12021

Simpson, M. R., Avershina, E., Storrø, O., Johnsen, R., Rudi, K., and Øien, T. (2018). Breastfeeding-associated microbiota in human milk following supplementation with Lactobacillus rhamnosus GG, Lactobacillus acidophilus La-5, and Bifidobacterium animalis ssp. lactis Bb-12. J. Dairy Sci. 101, 889-899. doi: $10.3168 /$ jds.2017-13411

Singh, D., and Fulekar, M. H. (2010). Biodegradation of petroleum hydrocarbons by Pseudomonas putida strain MHF 7109. Clean Soil Air Water 38, 781-786. doi: 10.1002/clen.200900239

Soeorg, H., Metsvaht, T., Eelmäe, I., Merila, M., Treumuth, S., Huik, K., et al. (2017). The role of breast milk in the colonization of neonatal gut and skin with coagulase-negative staphylococci. Pediatr. Res. 82, 759-767. doi: 10.1038/ pr.2017.150

Sørensen, U. B. S., Yao, K., Yang, Y., Tettelin, H., and Kilian, M. (2016). Capsular polysaccharide expression in commensal Streptococcus species: genetic and antigenic similarities to Streptococcus pneumoniae. MBio 7, 1844-1816e.

Summers, R. M., Mohanty, S. K., Gopishetty, S., and Subramanian, M. (2015). Genetic characterization of caffeine degradation by bacteria and its potential applications. Microbial Biotechnol. 8, 369-378. doi: 10.1111/1751-7915.12262

Treven, P., Mahnič, A., Rupnik, M., Golob, M., Pirš, T., Matijašić, B. B., et al. (2019). Evaluation of Human Milk Microbiota by 16S rRNA Gene Next-Generation Sequencing (NGS) and Cultivation/MALDI-TOF Mass Spectrometry Identification. Front. Microbiol. 10:2612. doi: 10.3389/fmicb.2019. 02612

Tuominen, H., Rautava, S., Collado, M. C., Syrjänen, S., and Rautava, J. (2018). HPV infection and bacterial microbiota in breast milk and infant oral mucosa. PLoS One 13:e0207016. doi: 10.1371/journal.pone.0207016

UNICEF (2018). "Infant and young child feeding". New York, NY: UNICEF.

Urbaniak, C., Angelini, M., Gloor, G. B., and Reid, G. (2016). Human milk microbiota profiles in relation to birthing method, gestation and infant gender. Microbiome 4:1.

Walker, A. (2010). Breast milk as the gold standard for protective nutrients. J. Pediatr. 156, S3-S7.

Wang, J., Wang, C., Li, J., Bai, P., Li, Q., Yuan, S. M., et al. (2018). Comparative genomics of degradative Novosphingobium strains with special reference to the 
microcystin-degrading Novosphingobium sp. THN1. Front. Microbiol. 9:2238. doi: $10.3389 /$ fmicb. 2018.02238

Ward, T. L., Hosid, S., Ioshikhes, I., and Altosaar, I. (2013). Human milk metagenome: a functional capacity analysis. BMC Microbiol. 13:116. doi: 10. 1186/1471-2180-13-116

Wei, T., and Simko, V. (2017). corrplot: Visualization of a Correlation Matrix. Vienna: R Core Team.

Weiss, S., Xu, Z. Z., Peddada, S., Amir, A., Bittinger, K., Gonzalez, A., et al. (2017). Normalization and microbial differential abundance strategies depend upon data characteristics. Microbiome 5:27.

Williams, J. E., Carrothers, J. M., Lackey, K. A., Beatty, N. F., Brooker, S. L., Peterson, H. K., et al. (2019). Strong Multivariate Relations Exist Among Milk, Oral, and Fecal Microbiomes in Mother-Infant Dyads During the First Six Months Postpartum. J. Nutrit. 149, 902-914. doi: $10.1093 /$ jn/nxy299

Williams, J. E., Carrothers, J. M., Lackey, K. A., Beatty, N. F., York, M. A., Brooker, S. L., et al. (2017). Human milk microbial community structure is relatively stable and related to variations in macronutrient and micronutrient intakes in healthy lactating women. J. Nutr. 147, 1739-1748.

World Health Organization (2014). Global nutrition targets 2025: Breastfeeding Policy Brief. Geneva: WHO.

Wren, H. M., Solomons, N. W., Chomat, A. M., Scott, M. E., and Koski, K. G. (2015). Cultural determinants of optimal breastfeeding practices among indigenous Mam-Mayan women in the Western Highlands of Guatemala J. Hum. Lact. 31, 172-184. doi: 10.1177/0890334414560194

Yabuuchi, E., Yano, I., Oyaizu, H., Hashimoto, Y., Ezaki, T., and Yamamoto, H. (1990). Proposals of Sphingomonas paucimobilis gen. nov. and comb. nov., Sphingomonas parapaucimobilis sp. nov., Sphingomonas yanoikuyae sp. nov., Sphingomonas adhaesiva sp. nov., Sphingomonas capsulata comb, nov., and Two Genospecies of the Genus Sphingomonas. Microbiol. Immunol. 34, 99-119. doi: 10.1111/j.1348-0421.1990.tb00996.x

Zhang, X., Liu, Y., Lin, Y., Wang, L., Yao, S., Cao, Y., et al. (2017). Novosphingobium clariflavum sp. nov., isolated from a household product plant. Int. J. Syst. Evol. Microbiol. 67, 3150-3155. doi: 10.1099/ijsem.0.001803

Conflict of Interest: The authors declare that the research was conducted in the absence of any commercial or financial relationships that could be construed as a potential conflict of interest.

Copyright (C) 2021 Gonzalez, Brereton, Li, Lopez Leyva, Solomons, Agellon, Scott and Koski. This is an open-access article distributed under the terms of the Creative Commons Attribution License (CC BY). The use, distribution or reproduction in other forums is permitted, provided the original author(s) and the copyright owner(s) are credited and that the original publication in this journal is cited, in accordance with accepted academic practice. No use, distribution or reproduction is permitted which does not comply with these terms. 